\title{
Optical properties of wurtzite/zinc-blende heterostructures in GaN nanowires
}

\author{
G. Jacopin, ${ }^{1, a)}$ L. Rigutti, ${ }^{1}$ L. Largeau, ${ }^{2}$ F. Fortuna, ${ }^{3}$ F. Furtmayr,${ }^{4,5}$ F. H. Julien, ${ }^{1}$ \\ M. Eickhoff, ${ }^{5}$ and M. Tchernycheva ${ }^{1}$ \\ ${ }^{1}$ Institut d'Electronique Fondamentale, Université Paris Sud XI, UMR 8622 CNRS, 15 Rue Clemenceau, \\ 91405 Orsay, France \\ ${ }^{2}$ Laboratoire de Photonique et Nanostructures, UPR 20 CNRS, Route de Nozay, 91460 Marcoussis, France \\ ${ }^{3}$ Centre de Spectroscopie Nucléaire et Spectroscopie de Masse, Université Paris Sud XI, UMR 8609 CNRS, \\ 15 Rue Clemenceau, 91405 Orsay, France \\ ${ }^{4}$ Walter-Schottky-Institut, Technische Universität München, Am Coulombwall 3, 85748 Garching, Germany \\ ${ }^{5}$ I. Physikalisches Institut, Justus-Liebig-Universität, Heinrich-Buff-Ring 16, 35392 Giessen, Germany
}

(Received 5 May 2011; accepted 8 August 2011; published online 21 September 2011)

\begin{abstract}
The optical and structural properties of wurtzite GaN nanowires containing zinc-blende GaN inclusions of different thicknesses are investigated. Micro-photoluminescence spectra of single nanowires exhibit a series of narrow emission peaks with linewidth as low as $0.8 \mathrm{meV}$ in the interval $3.1-3.42 \mathrm{eV}$. The peak energy blue-shifts with increasing excitation power following a $\sim \mathrm{I}^{1 / 3}$ law due to the progressive band filling and to the screening of the internal field. The quantum confinement in these type-II crystal phase heterostructures was simulated in the framework of a one-dimensional effective mass model, accounting for the internal electrical polarization of the wurtzite $\mathrm{GaN}$. The predicted transition energies are in good agreement with the energy statistics realized on more than 30 single nanowire emission spectra. (C) 2011 American Institute of Physics. [doi:10.1063/1.3638698]
\end{abstract}

\section{INTRODUCTION}

The development of semiconductor nanowires grown by molecular beam epitaxy (MBE) or by metal-organic chemical vapour deposition has opened up the possibility to design novel devices based on either compositional or crystal phase heterostructures. Axial crystal phase heterostructures consisting of adjacent nanowire segments of different polytypes have been the object of dedicated theoretical and experimental studies. ${ }^{1-7}$ The most common crystal phase heterostructure consists of hexagonal wurtzite (WZ) and cubic zincblende (ZB) polytypes. These heterostructures are typical of type-II, i.e., a ZB inclusion inside a WZ matrix forms a quantum well confining electrons, whereas a WZ inclusion in a $\mathrm{ZB}$ matrix forms a quantum well confining holes. This arrangement has no counterpart in the bulk materials, where inclusions of a different crystal polytypes inside a 3D matrix cannot be controlled and are typically surrounded by dislocations. In nanowires, crystal-phase heterostructures present three main advantages with respect to compositional ones: (1) the abruptness of the heterointerface, coinciding with a single basal plane, (2) chemical homogeneity, and (3) the low lattice mismatch. So far, crystal phase heterostructures have been demonstrated in classic III-V nanowires (mostly InP, InAs, or GaAs). Optical studies have proven the type-II alignment ${ }^{4}$ and have demonstrated narrow line, ${ }^{5,6}$ and even single-photon emission. ${ }^{3}$ In parallel, significant efforts have been made in order to achieve a sufficient growth control for the realization of well defined superlattices. ${ }^{2}$ Recently, the formation of WZ/ZB heterostructures has also been reported in MBE-grown II-VI nanowire systems. ${ }^{8}$ For the group

\footnotetext{
a) Author to whom correspondence should be addressed. Electronic mail gwenole.jacopin@ief.u-psud.fr.
}

III-nitride material system reports of WZ/ZB heterostructures have not been published so far, with the only exception of the work by Renard et al. ${ }^{9}$ analyzing the properties of $\mathrm{GaN}$ nanowires with a $\mathrm{WZ}$ base and a $\mathrm{ZB}$ top segment. In $\mathrm{GaN}$, the properties of the $\mathrm{ZB}$ phase ${ }^{10}$ of its inclusions in a WZ matrix ${ }^{11}$ and of stacking faults (SF) (Refs. 12-14) have been studied in the bulk material. Both theoretical ${ }^{15,16}$ and experimental $^{11,14}$ studies have shown that the band alignment between the $\mathrm{WZ}$ and $\mathrm{ZB}$ phases in nitrides is type-II. Basal SFs can be regarded as a single cubic sequence of planes $(\mathrm{ABC})$ in the hexagonal matrix $(\mathrm{ABAB} \ldots)$ and are associated with a strong luminescence signal due to bound excitons at energy $3.41-3.42 \mathrm{eV}^{12-14}$ In the case of GaN nanowires, however, the presence of $\mathrm{ZB}$ inclusions of different thickness in WZ GaN has only been reported for heavily Mg-doped NWs (Ref. 17) and an optical analysis of such structures has not been carried out.

In this work, we analyze the optical properties of $\mathrm{ZB} /$ WZ heterostructures in GaN nanowires. We demonstrate that these heterostructures give rise to excitonic transitions with a narrow linewidth. The nanowires have been grown using plasma-assisted molecular beam epitaxy (PA-MBE), structurally analyzed by scanning transmission electron microscopy (STEM) and optically studied by microphotoluminescence ( $\mu$-PL). STEM analyses reveal that ZB inclusions of 2 to 10 monolayer (ML) thickness are present in the WZ matrix of the nanowires. The spectra of single nanowires exhibit a serie of emission peaks with linewidth as low as $0.8 \mathrm{meV}$ in the range of $3.1 \mathrm{eV}-3.42 \mathrm{eV}$. These peaks blue-shift with increasing excitation power due to the progressive band filling and to the screening of the internal field. We interpret our observations by means of a $1 D$ effective mass model, simulating the $\mathrm{WZ} / \mathrm{ZB} / \mathrm{WZ}$ heterostructure as a type-II quantum well and accounting for the internal 
electrical polarization of WZ GaN. The model well illustrates the dependence of the PL energy on the thickness of the $\mathrm{ZB}$ inclusion.

\section{SAMPLE STRUCTURE}

GaN NWs with a length of approximately $300 \mathrm{~nm}$ and a diameter ranging between $25 \mathrm{~nm}$ and $30 \mathrm{~nm}$ were grown by PA-MBE on n-type $\mathrm{Si}(111)$ substrates at a substrate temperature of $775^{\circ} \mathrm{C}$ using nitrogen rich growth conditions according to the procedure described in Ref. 18. On top of the GaN base part an $\mathrm{InGaN}$ section with a nominal In-concentration of approximately $10 \%$ and a length of $100 \mathrm{~nm}$ was grown at a reduced substrate temperature of $590^{\circ} \mathrm{C}$, followed by 9 periods of alternating regions of $\mathrm{GaN}(7 \mathrm{~nm})$ and $\mathrm{InGaN}$ $(2 \mathrm{~nm})$ and a $\mathrm{GaN}$ cap layer with a nominal thickness of $25 \mathrm{~nm}$ at the same substrate temperature. A gradual increase of the NW diameter from $50 \mathrm{~nm}$ to $80 \mathrm{~nm}$ is observed in the InGaN and InGaN/GaN region deposited at lower substrate temperatures.

The nanowire structure was first analyzed by STEM revealing the presence of stacking faults in the GaN cap, which was found to be longer than the nominal value $(\sim 50$ $\mathrm{nm}$ ). The bright-field STEM images in Fig. 1 depict a region close to the top of one nanowire, with the viewing direction parallel to the $\langle 1-210\rangle$ crystal axis. In Fig. 1(a) several ZB inclusions of different thicknesses are visible. Several of them are indicated by arrows. An energy-dispersive x-ray spectroscopy (EDX) analysis performed in STEM showed no presence of In in this region. As reported in other III-V nanowire systems containing WZ/ZB heterostructures, ${ }^{3,5}$ the interfaces between the different crystal phases coincide perfectly with basal atomic planes. The high-resolution detail shown in Fig 1(b) is a high magnification image of one selected region where $\mathrm{ZB}$ inclusions of 2, 3, and 6 MLs are present. It is worth noticing that the two inclusions in the lower part of Fig. 1(b) are separated only by one single monolayer violation of the cubic stacking sequence.

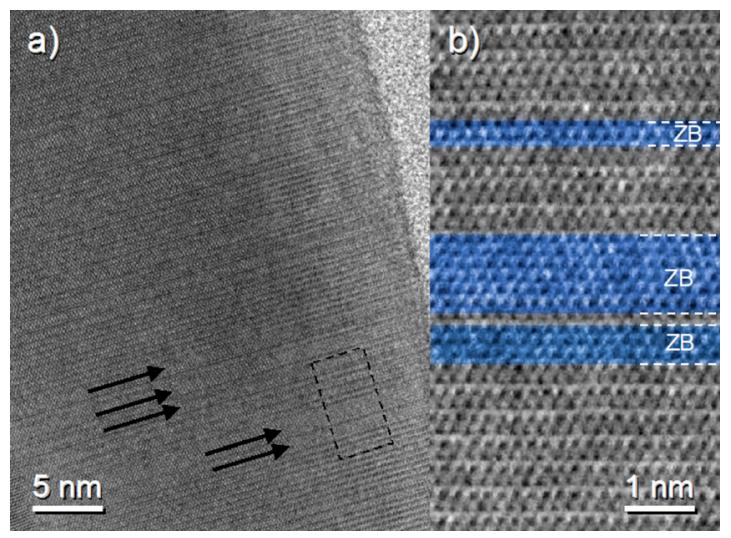

FIG. 1. (Color online) (a) STEM image of one nanowire from the analysed sample. The arrows indicate the position of several ZB inclusions. The dashed rectangle indicates the region depicted in the high-resolution detail of part (b), where ZB inclusions of $2 \mathrm{ML}, 6 \mathrm{ML}$, and $3 \mathrm{ML}$ are evidenced in blue and delimited by the white dashed lines.

\section{OPTICAL PROPERTIES}

For the $\mu$-PL studies, nanowires were detached from the substrate by ultrasound bath in ethanol, and dispersed on $\mathrm{Si}$ substrates. The nanowire density was controlled in the range of $1-5 \times 10^{6} \mathrm{~cm}^{-2}$. The $\mu$-PL characterization was carried out at $\mathrm{T}=4.2 \mathrm{~K}$ by exciting the sample with $\mathrm{cw} 244 \mathrm{~nm}$ light from a frequency-doubled $\mathrm{Ar}^{++}$ion laser. The laser radiation was focused onto the substrate surface with a spot of diameter $\sim 5 \mu \mathrm{m}$ by means of a $20 \times \mathrm{UV}$ microscope objective with a numerical aperture of 0.4 . The excitation power was varied between $2.5 \mu \mathrm{W}$ and $1 \mathrm{~mW}$. The luminescence was collected with the same objective, dispersed with a $460 \mathrm{~mm}$ focal length spectrometer and detected with a charge coupled device with a spectral resolution of $500 \mu \mathrm{eV}$.

Figure 2(a) presents a photoluminescence spectrum of the nanowire ensemble showing two main contributions at energies 2.4-2.7 eV and at 3.1-3.42 eV. An example of a $\mu$-PL spectrum of a single nanowire shown in Fig. 2(b) exhibits narrow emission peaks distributed in the same energy intervals as for the ensemble. The emission in the 2.4-2.7 eV interval is attributed to the luminescence of the InGaN quantum discs (QDs) and of the InGaN segment. The second high-energy contribution is attributed to the presence of $\mathrm{WZ} / \mathrm{ZB}$ heterostructures in the GaN nanowire top region. The GaN near-band edge emission (3.45-3.48 eV) has been observed in several nanowires, but it is systematically very weak compared to the other spectral contributions. In the following, the discussion will concentrate on the narrow emission lines found in the range of 3.1-3.42 eV.

Figure 2(c) shows other examples of single nanowire spectra exhibiting multiple sharp lines with full width at half maximum $(\mathrm{FWHM})<5 \mathrm{meV}$ in the range of $3.1-3.42 \mathrm{eV}$. Some of these emissions are very intense and extremely narrow. The inset shows a close-up and the Lorentzian fits of a selected spectral region exhibiting sharp peak emission. The peaks marked as 1,2, and 3 have a linewidth in the range of

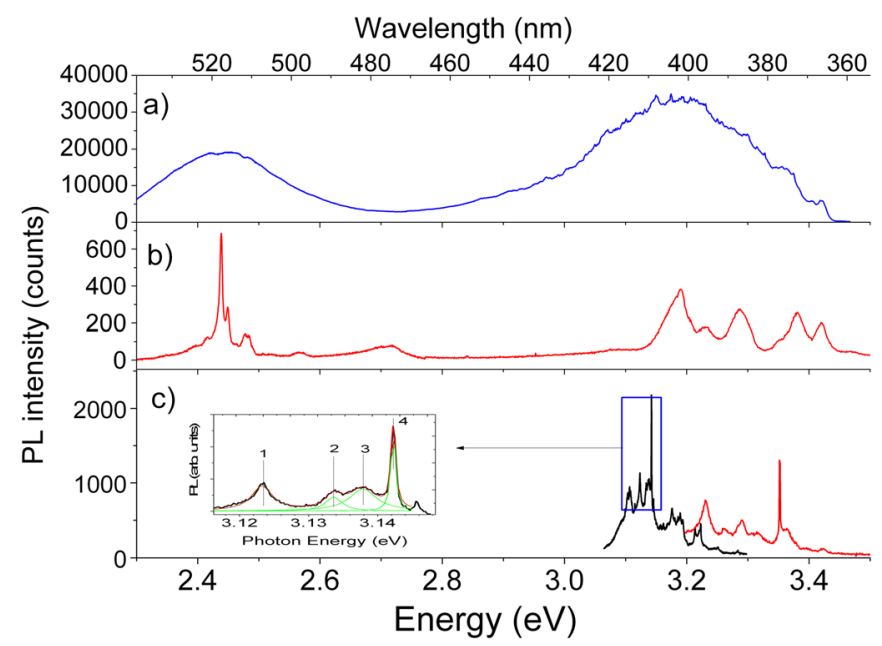

FIG. 2. (Color online) (a) PL spectrum of the nanowire ensemble at $4 \mathrm{~K}$. (b) $\mu$-PL spectrum of a single nanowire at $4 \mathrm{~K}$ (b) $\mu$-PL spectra of two nanowires exhibiting narrow emissions related to WZ/ZB heterostructures. The inset shows a detail of one spectrum and the Lorentzian fits of selected peaks. The energy and FWHM of the narrowest peak are $\mathrm{E}_{4}=3.1423 \mathrm{eV}$ and $\Gamma_{4}=0.8 \mathrm{meV}$. 
2-3 meV, while peak 4 at $\mathrm{E}_{4}=3.1423 \mathrm{eV}$ has a FWHM of 0.8 $\mathrm{meV}$-close to the state of the art broadening achieved in Stranski-Krastanov GaN quantum dots. ${ }^{19}$ It should be noted that in the case of crystal phase quantum heterostructures, the lineshape is well reproduced with a Lorentzian fit contrary to what is observed in GaN/AlN single quantum dots, where the lineshape is mainly Gaussian due to the spectral diffusion. ${ }^{20}$

The power dependence of the luminescence peaks is exemplified in Fig. 3. When the power intensity is progressively increased over two decades, the peak blue shifts and broadens (Fig. 3(a)). The blue shift can be well reproduced by a cubic root law $E(I)-E_{0} \sim I^{1 / 3}$, where $E$ is the peak energy, $I$ is the laser intensity, and $E_{0}=3.283 \mathrm{eV}$ is the peak energy value extrapolated for small excitation power. The peak energy varies by $30 \mathrm{meV}$ in two decades of excitation intensity. The integrated PL intensity of the peak $S_{P L}$ grows slightly sub-linearly with the excitation power, as $S_{P L} \sim I^{0.8}$.

\section{DISCUSSION}

The blue-shift with increasing excitation power suggests a relation with the $\mathrm{WZ} / \mathrm{ZB}$ type-II heterostructure, as observed in InP and GaAs nanowires. ${ }^{3,5,6}$ The blue shift can be interpreted as the result of field screening, consequence of the relatively slow recombination of the photogenerated electron-hole pairs. This effect is typical of type-II heterostructures, while in type-I heterostructures, the exciton transition energy is independent of the excitation power. ${ }^{21}$

In type-II heterostructures the e-h subbands involved in the transitions tend to progressively populate when the exci-
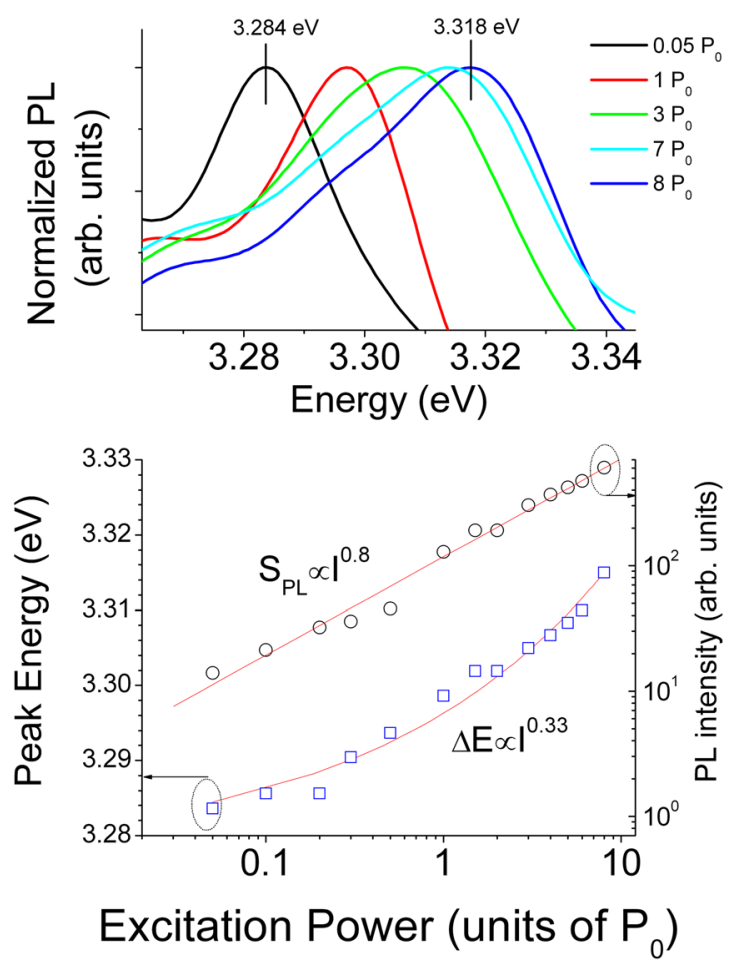

FIG. 3. (Color online) (a) Normalized PL of a selected peak from a single nanowire exhibiting a progressive blue shift with increasing excitation power. (b) Dependence of peak energy (blue empty square) and peak intensity (black circles) on the excitation power. $\mathrm{P}_{0}=50 \mu \mathrm{W}$ is the laser power transmitted by the $\mu$-PL objective. tation power is raised, thus blue-shifting the PL energy and broadening the peak. The band filling also leads to the formation of an electric dipole across the WZ/ZB interface, which partially screens the internal polarization field and contributes to the blue shift of the transition by counteracting the quantum confined Stark effect (QCSE). Taking these effects into account, the dependence of the blue shift of PL transition on the excitation power can be modeled as a cubic root law in type II heterostructure systems such as GaAsSb/GaAs multiQWs (Ref. 22) and InAs/GaAs QDs covered by GaAsSb. ${ }^{23} \mathrm{~A}$ slight deviation from the cubic root dependence in the case illustrated in Fig. 3 might be explained by a possible overlap of two peaks originating from two different regions. The first one related to a single type II heterostructure, which follows the cubic root law. The second one is related to a coupled quantum well less sensitive to power excitation at $3.295 \mathrm{eV}$.

It should be noted that a different power behavior was found by Bao et al. ${ }^{4}$ in the study of the PL in InP nanowires containing ZB/WZ type-II superlattices. For InP, the power dependence of the blue shift is almost linear with the logarithm of the excitation power contrary to the cubic root law found in the present case. However, those heterostructures are characterized by a lower band offset and negligible internal polarization fields, ${ }^{24}$ which can explain the difference with the GaN case. Furthermore, not all peaks observed in the present study follow the same power behavior, the blue shift being extremely reduced (a few meV) in about $30 \%$ of the cases. We suppose that these peaks with reduced blueshift might be related to a different mechanism, such as recombination of an exciton localized at a defect or a donoracceptor pair (DAP) transition. Indeed, it should be noticed that the spectral interval where the narrow peaks appear in the present samples overlaps with the DAP band in $\mathrm{WZ}$ and $\mathrm{ZB}$ $\mathrm{GaN}(\mathrm{E} \sim 3.0-3.3 \mathrm{eV}),{ }^{10,25,26}$ which has also been reported in nanowires. ${ }^{18,27-29}$ However, the attribution of the narrow peaks in the $3.1 \mathrm{eV}-3.42 \mathrm{eV}$ range to DAP seems to be inappropriate in the present case. The DAP emission is indeed characterized by a very different spectral shape in GaN thin film and nanowire samples, ${ }^{18,25,26,28,29}$ where the two dominant emissions are at $3.21 \mathrm{eV}$ and $3.27 \mathrm{eV}$ and are significantly broader than the features found in the present samples.

\section{MODELING}

The optical properties of the analyzed structures were compared to a one-dimensional simulation in the framework of the effective mass approximation. ${ }^{30}$ The SchrödingerPoisson equation was solved self-consistently in the type-II quantum well where the $\mathrm{ZB}$ inclusion is embedded in a $\mathrm{WZ}$ matrix. For this quantum well, the conduction and valence band discontinuities are $\Delta \mathrm{E}_{\mathrm{c}}=270 \mathrm{meV}$ and $\Delta \mathrm{E}_{\mathrm{v}}=70 \mathrm{meV}$, respectively. ${ }^{14,16}$ The effective masses of electrons and heavy holes in the $\mathrm{WZ}$ and $\mathrm{ZB}$ phases are $\mathrm{m}_{\mathrm{e}, \mathrm{WZ}}^{*}=0.2 \mathrm{~m}_{0}$, $\mathrm{m}_{\mathrm{e}, \mathrm{ZB}}^{*}=0.15 \mathrm{~m}_{0}, \mathrm{~m}_{\mathrm{hh}, \mathrm{WZ}}^{*}=1.4 \mathrm{~m}_{0}$, and $\mathrm{m}_{\mathrm{hh}, \mathrm{ZB}}^{*}=1.3 \mathrm{~m}_{0}{ }^{31}$ The excitonic binding energy was neglected (this energy is expected to be small in type II heterostructures). It should be noted that these simulations provide an estimation explaining the trend of photoluminescence energies when the number of 
thickness of ZB inclusions increases rather than a rigorous calculation of energy of the photoluminescence lines.

The spontaneous polarization of the WZ phase $\mathrm{P}=0.029 \mathrm{C} / \mathrm{m}^{2}$ is taken into account, ${ }^{31}$ yielding a discontinuity of the electric field across the WZ/ZB interface $\Delta \mathrm{F}=1.8 \mathrm{MV} / \mathrm{cm}$. The residual doping level was assumed as $\mathrm{N}_{\mathrm{d}}=10^{17} \mathrm{~cm}^{-3}$. To calculate the transition energies, the coupling of a single WZ/ZB/WZ crystal phase sequence with surrounding $\mathrm{ZB}$ or $\mathrm{WZ}$ regions was neglected. Figure 4(a) depicts the simulation results for a narrow $\mathrm{ZB}$ inclusion of 3 $\mathrm{ML}$ and for a larger ZB inclusion of $7 \mathrm{ML}$, while the resulting PL transition energies of the $\mathrm{e}_{1}-\mathrm{hh}_{1}$ subbands calculated for ZB inclusions of thickness from 2 ML (basal stacking fault) to $9 \mathrm{ML}$ are reported in Fig. 4(b) as vertical dotted lines. The sketch in Fig. 4(a) underlines the importance of the spontaneous polarization field of the WZ phase, yielding a triangular shape of the potentials confining electrons and holes on the two sides of the WZ/ZB interface. The electric field enhances the red shift of the PL energies with increasing thickness of the $\mathrm{ZB}$ inclusion. The transition energy falls below the bulk ZB-GaN bandgap ${ }^{9,10}$ when the thickness of the $\mathrm{ZB}$ inclusion is at least $6 \mathrm{ML}$ thick (indicated as the cubic bandgap in Fig. 4(b)). On average, the emission energy red

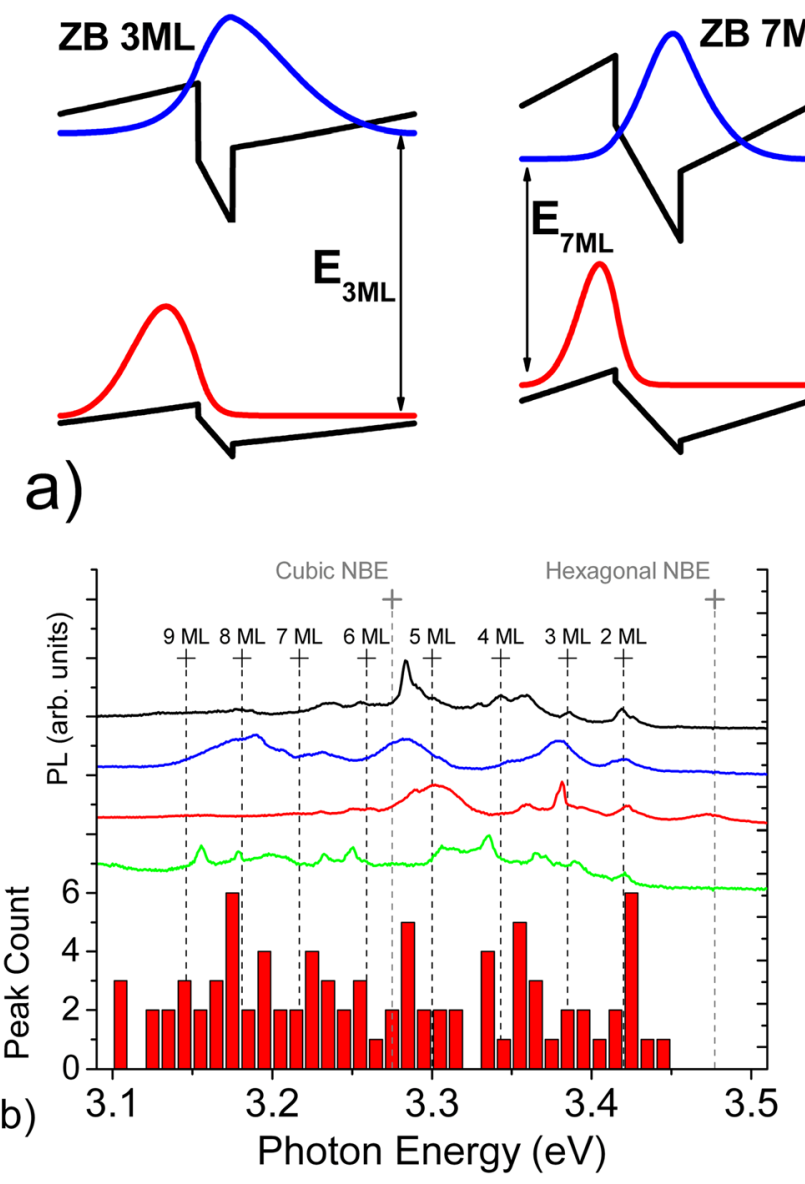

FIG. 4. (Color online) (a) $1 \mathrm{D}$ effective mass simulation of the band diagram and $\mathrm{e}-\mathrm{h}$ wavefunctions for a $\mathrm{ZB}-\mathrm{GaN}$ inclusion of 3 atomic monolayers (left) and 7 atomic monolayers (right). (b) Sample $\mu$-PL spectra from dispersed nanowires and statistics of the peak energies. The experimental data are compared to the simulation of ZB inclusions of different thickness in the WZ matrix (black dashed vertical lines) and to the near band edge emissions of bulk ZB and WZ GaN (grey dotted lines). shifts by $30-40 \mathrm{meV}$ for one additional ZB ML due to the contribution of both quantum confinement and in particular the quantum confined Stark effect. A much smaller value of around $10 \mathrm{meV}$ is found in the case of $\mathrm{ZB} / \mathrm{WZ}$ type II quantum wells in InP nanowires, ${ }^{6}$ where the band offsets are $\Delta \mathrm{E}_{\mathrm{c}}=129 \mathrm{meV}$ and $\Delta \mathrm{E}_{\mathrm{v}}=45 \mathrm{meV}$ (Ref. 32) and where the spontaneous polarization of the $\mathrm{WZ}$ phase is negligible.

In Fig. 4(b), the calculated values of the transition energies are compared to four sample $\mu$-PL spectra and to the statistics of the peak energies occurring in around 30 single nanowire spectra. The spectra and the statistics show that there is a reasonable agreement between the predictions of the model and the experimental peak energies. In particular, the increase of the peak occurrence is found in correspondence of the predicted transition energy for the $\mathrm{ZB}$ inclusion of $2 \mathrm{ML}, 4 \mathrm{ML}, 5 \mathrm{ML}, 7 \mathrm{ML}$, and $8 \mathrm{ML}$. This behavior is not expected for DAP transitions, which either give rise to several broad peaks or to a continuous broad band. ${ }^{25,26}$ The intensity of the emission lines is determined both by the oscillator strength of the corresponding transition and by the number of the cubic inclusions with the given thickness in the nanowire. However, it must be kept in mind that other effects can influence the transition intensity and energies. Among them, the possibility of local potential variations due to the distribution of impurities ${ }^{14}$ or the coupling between neighboring $\mathrm{ZB}$ inclusions, which explains the presence of peaks with intermediate energies. Indeed, for narrow $\mathrm{ZB}$ inclusions the confined electron wave function strongly extends into the WZ barriers, as can be also seen in Fig. 4(a), and couples with neighboring ZB inclusions. Strong coupling, for instance, is expected for ZB inclusions such as those reported in Fig. 1(b), separated by only one violation of the cubic stacking sequence. These two effects naturally lead to further spreading of the energy levels, which are experimentally reflected by the dispersion of the peak energy statistics in between the calculated transition energies.

\section{CONCLUSIONS}

In summary, we have shown that the $\mu$-PL spectra of single GaN nanowires containing type-II WZ/ZB heterostructures exhibit excitonic emissions with linewidth as low as $0.8 \mathrm{meV}$. The behavior of the peaks as a function of the excitation power suggests a relation with the presence of type-II heterostructures. We studied the dependence of the emission energy on the heterostructure parameters in the framework of a $1 D$ effective mass simulation, where we modeled the transition in the type-II WZ/ZB/WZ quantum well. The transition energies predicted by the simulation for ZB inclusions of varying ML thickness are in good correlation with the statistics realized on more than 30 single nanowire spectra, strongly supporting the attribution of the observed narrow peaks to excitonic transitions occurring in the WZ-ZB type-II heterostructures.

\section{ACKNOWLEDGMENTS}

This work was supported by the French ANR agency under the programs ANR-08-NANO-031 BoNaFo and ANR08-BLAN-0179 NanoPhotoNit. 
${ }^{1}$ F. Glas, J.-C. Harmand, and G. Patriarche, Phys. Rev. Lett. 99, 3 (2007).

${ }^{2}$ P. Caroff, K. A. Dick, J. Johansson, M. E. Messing, K. Deppert, and L. Samuelson, Nat. Nanotechnology 4, 50-55 (2009).

${ }^{3}$ N. Akopian, G. Patriarche, L. Liu, J.-C. Harmand, and V. Zwiller, Nano Lett. 10, 1198 (2010)

${ }^{4}$ J. Bao, D. C. Bell, F. Capasso, J. B. Wagner, T. Mårtensson, J. Trägårdh, and L. Samuelson, Nano Lett. 8, 836 (2008).

${ }^{5}$ D. Spirkoska, J. Arbiol, A. Gustafsson, S. Conesa-Boj, F. Glas, I. Zardo, M. Heigoldt, M. H. Gass, A. L. Bleloch, S. Estrade, M. Kaniber, J. Rossler, F. Peiro, J. R. Morante, G. Abstreiter, L. Samuelson, and A. Fontcuberta i Morral, Phys. Rev. B 80, 1 (2009).

${ }^{6}$ J.-M. Jancu, K. Gauthron, L. Largeau, G. Patriarche, J.-C. Harmand, and P. Voisin, Appl. Phys. Lett. 97, 041910 (2010).

${ }^{7}$ I. Zardo, S. Conesa-Boj, F. Peiro, J. R. Morante, J. Arbiol, E. Uccelli, G. Abstreiter, and A. Fontcuberta i Morral, Phys. Rev. B 80, 1 (2009).

${ }^{8}$ E. Bellet-Amalric, M. Elouneg-Jamroz, C. Bougerol, M. Den Hertog, Y. Genuist, S. Bounouar, J. P. Poizat, K. Kheng, R. André, and S. Tatarenko, Phys. Status Solidi C 7, 1526 (2010).

${ }^{9}$ J. Renard, G. Tourbot, D. Sam-Giao, C. Bougerol, B. Daudin, and B. Gayral, Appl. Phys. Lett. 97, 081910 (2010).

${ }^{10}$ D. J. As, F. Schmilgus, C. Wang, B. Schottker, D. Schikora, and K. Lischka, Appl. Phys. Lett. 70, 1311 (1997).

${ }^{11}$ X. H. Lu, P. Y. Yu, L. X. Zheng, S. J. Xu, M. H. Xie, and S. Y. Tong, Appl. Phys. Lett. 82, 1033 (2003).

${ }^{12}$ G. Salviati, M. Albrecht, C. Zanotti-Fregonara, N. Armani, M. Mayer, Y. Shreter, M. Guzzi, Yu. V. Melnik, K. Vassilevski, V. A. Dmitriev, and H. P. Strunk, Phys. Status Solidi A 171, 325 (1999).

${ }^{13}$ R. Liu, A. Bell, F. A. Ponce, C. Q. Chen, J. W. Yang, and M. A. Khan, Appl. Phys. Lett. 86, 021908 (2005).

${ }^{14}$ P. Corfdir, P. Lefebvre, J. Ristić, P. Valvin, E. Calleja, A. Trampert, J.-D. Ganière, and B. Deveaud-Plédran, J. Appl. Phys. 105, 013113 (2009).

${ }^{15}$ Z. Bandić, T. McGill, and Z. Ikonić, Phys. Rev. B 56, 3564 (1997).

${ }^{16}$ C. Stampfl and C. G. de Walle, Phys. Rev. B 57, R15052 (1998).
${ }^{17}$ J. Arbiol, S. Estradé, J. D. Prades, A. Cirera, F. Furtmayr, C. Stark, A. Laufer, M. Stutzmann, M. Eickhoff, M. H. Gass, A. L. Bleloch, F. Peiró, and J. R. Morante, Nanotechnology 20, 145704 (2009).

${ }^{18}$ F. Furtmayr, M. Vielemeyer, M. Stutzmann, A. Laufer, B. K. Meyer, and M. Eickhoff, J. Appl. Phys. 104, 074309 (2008).

${ }^{19}$ D. Simeonov, A. Dussaigne, R. Butté, and N. Grandjean, Phys. Rev. B 77, 1 (2008).

${ }^{20}$ F. Demangeot, D. Simeonov, A. Dussaigne, R. Butté, and N. Grandjean, Phys. Status Solidi C 6, S598 (2009).

${ }^{21}$ L. Rigutti, M. Tchernycheva, A. De Luna Bugallo, G. Jacopin, F. H. Julien, F. Furtmayr, M. Stutzmann, M. Eickhoff, R. Songmuang, and F. Fortuna, Phys. Rev. B 81, 045411 (2010).

${ }^{22}$ Y. S. Chiu, M. H. Ya, W. S. Su, and Y. F. Chen, J. Appl. Phys. 92, 5810 (2002).

${ }^{23}$ C. Y. Jin, H. Y. Liu, S. Y. Zhang, Q. Jiang, S. L. Liew, M. Hopkinson, T. J. Badcock, E. Nabavi, and D. J. Mowbray, Appl. Phys. Lett. 91, 021102 (2007).

${ }^{24}$ M. Heiss, S. Conesa-Boj, J. Ren, H.-H. Tseng, A. Gali, A. Rudolph, E. Uccelli, F. Peiró, J. Morante, D. Schuh, E. Reiger, E. Kaxiras, J. Arbiol, and A. Fontcuberta i Morral, Phys. Rev. B 83, 1 (2011).

${ }^{25}$ L. Eckey, J.-Ch. Holst, P. Maxim, R. Heitz, A. Hoffmann, I. Broser, B. K. Meyer, C. Wetzel, E. N. Mokhov, and P. G. Baranov, Appl. Phys. Lett. 68, 415 (1996).

${ }^{26}$ M. Reshchikov, G.-C. Yi, and B. Wessels, Phys. Rev. B 59, 13176 (1999).

${ }^{27}$ E. Calleja, M. Sánchez-García, F. Sánchez, F. Calle, F. Naranjo, E. Muñoz, U. Jahn, and K. Ploog, Phys. Rev. B 62, 16826 (2000).

${ }^{28}$ H. Y. Chen, H. W. Lin, C. H. Shen, and S. Gwo, Appl. Phys. Lett. 89, 243105 (2006).

${ }^{29}$ J. B. Schlager, K. A. Bertness, P. T. Blanchard, L. H. Robins, A. Roshko, and N. A. Sanford, J. Appl. Phys. 103, 124309 (2008).

${ }^{30}$ Nextnano, see nextnano website http://www.wsi.tum.de/nextnano3 for tutorial input files and detailed documentation.

${ }^{31}$ I. Vurgaftman, J. R. Meyer, and L. R. Ram-Mohan, J. Appl. Phys. 89, 5815 (2001).

${ }^{32}$ M. Murayama and T. Nakayama, Phys. Rev. B 49, 4710 (1994). 